\title{
Endophyte survival without sex
}

\author{
B.R. HOLLAND ${ }^{1}$, C.L. SCHARDL ${ }^{2}$ and J. SCHMID ${ }^{3}$ \\ ${ }^{I}$ Institut e of Fundamental Sciences, Massey Univerisity, NZ \\ ${ }^{2}$ University of Kentucky, Department of Plant Pathology, 201 PSB, 1405 Veterans Dr., Lexington, KY 40546-0312, U.S.A. \\ ${ }^{3}$ Institute of Molecular BioSciences, Massey University, NZ \\ j.schmid@massey.ac.nz
}

\begin{abstract}
Sex is considerably more costly for an organism than clonal reproduction. How it conveys sufficient benefits to outweigh this cost remains unclear. One of the main arguments that such benefits exist is the wide distribution of sex and the short evolutionary lifespan of asexual lineages. However, too little is known about the reproductive biology of microorganisms to be certain that sex is wide-spread among them. Among the epichloë endophytes the ability to carry out sexual reproduction is frequently lost, and closely related sexual and asexual lineages can be readily collected. This offers an opportunity to test if asexual endophyte lineages have a shorter lifespan than sexual lineages, which would indicate that sex conveys advantages to epichloë endophytes. Using a novel phylogeny-based statistical approach, we found strong evidence that asexual endophyte lineages are short-lived compared to sexual lineages.
\end{abstract}

Keywords: sex, clonal reproduction, evolutionary biology, ecology

\section{Introduction}

Why sex ever evolved and has survived as a reproductive strategy remains one of the great mysteries of biology. It is well established that sex is more costly than clonal reproduction. How it conveys sufficient benefits to outweigh this cost remains unclear. Attempts to answer this question have merely confirmed that sex is costly and that even the mixing of genetic material, proposed as its main compensating benefit, is detrimental in most situations (Goddard et al. 2005; Otto \& Lenormand 2002; Rice 2002).

Nevertheless, biologists generally consider sex to be superior to clonal reproduction, because it is wide-spread, and because asexual lineages are usually short-lived (Otto \& Lenormand 2002; Rice 2002). However, this conclusion is based largely on macroscopic species -plants and animals- with comparatively small population sizes. Our knowledge of the reproductive strategies of microscopic organisms is scant, and some of them are believed to have survived for long periods without sex, such as the Glomales (arbuscular mycorrhizal fungi) and the bdelloid rotifers (bacterial unidirectional transfer of genetic material is a form of sex, and thus bacteria are not necessarily clonal organisms) (Carlile et al. 2001; Goddard et al. 2005; Maynard Smith et al. 1993; Otto \& Lenormand 2002; Purvis \& Hector 2000; Rice 2002; Tibayrenc 1999; Welch \& Meselson 2000). It is thus still an open question if sex is universally widespread and if asexual lineages are generally short-lived.

Among the fungal epichloë endophytes of grasses asexual reproduction is wide-spread, suggesting that it conveys advantages over sex at least in the short term, although many instances of hybridisation among asexual species suggest that rare exchange of genetic material may benefit these otherwise asexual lineages (Moon et al. 2004). However, it has not yet been rigorously tested if endophytes can survive in the long run (on an evolutionary scale) without sex or hybridisation. This paper describes an investigation into the long-tem survival of asexual endophytes.

\section{Methods}

In all, 32 tef $A$ and $33 t u b B$ sequences were selected from a larger dataset (Gentile et al. 2005; Moon et al. 2004) to create a data set for each gene with no identified hybrids and only one instance of each species (Table 1; where the level of divergence between two taxa that were supposedly of the same species was higher than that between pairs of taxa in different species taxa, the divergent sequences were retained). Phylogenetic analysis was carried out using MrBayes (version 3.1.2) to sample from the space of all possible trees in proportion to their probability given the data. The trees were forced to be clock-like, so that the distance from the root to the tip was equal for all taxa. The software was run for 150000 generations and a tree was sampled every $100^{\text {th }}$ generation. The first 200 sampled trees were discarded as part of the burn-in, resulting in a sample of 2600 trees for each gene. For each tree we inferred the state - sexual or asexual - at the internal nodes by assigning any internal node with two asexual descendents the asexual state and any other internal node the sexual state. Then for each asexual species in each of the 2600 trees we calculated the distance to the most ancient ancestral node that can be reached by a path that never passes through a sexual node plus half the branch length of the branch leading to the first sexual ancestor. To find the null distribution of this statistic, we used a permutation test that randomly allocated the asexual and sexual states to each species, and for each random allocation, we recalculated the test statistic.

\section{Results}

To determine if asexual endophytes survive for as long as sexual species, we wanted to use evolutionary trees in which we would determine how far back in time we can trace asexual ancestors of extant asexual species. We can calculate the time of survival of an asexual lineage as the time that has elapsed between the present and the time of existence of its most ancient asexual ancestor, connected to the extant strains by an unbroken series of asexual intermediates. The ancestor and intermediates themselves may be extinct, but we can infer their reproductive mode sexual or asexual from the tree: it seems reasonable to assume that accumulation of multiple mutations in the sexual machinery following the initial loss of a sexual cycle would make it very difficult to restore sex - reversion of each of these mutations would in itself not reconstitute a sexual cycle and would not convey a selective advantage. Therefore, we can assume that all branch-points in a tree which lead only to extant asexual species represent asexual ancestors. In contrast, all branch-points that lead to extant sexual species or to both asexual and sexual species represent sexual ancestors (Fig. 1). We can then compare these survival times to the time of survival of sexual lineages. However we cannot do this directly because sex is ancient (Otto \& Lenormand 2002; Rice 2002) and the oldest ancestor of all extant endophyte lineages is therefore sexual. Because of this, sexual lineages should on average have a longer lifespan, even if the chances of survival of a lineage, once sex has been lost, is equal to that of a sexual lineage. To overcome this problem we 
Table 1 Endophyte isolates and their tubB and tef $A$ sequences.

GenBank accessions

\begin{tabular}{|c|c|c|c|c|}
\hline Species* & Designation* & Host & tubB & tefA \\
\hline Epichloë amarillans & Eama200743 & Agrostis hiemalis & L06959 & AF231192 \\
\hline E. baconii & Ebab76552 & Agrostis stolonifera & L06961 & AF231193 \\
\hline E. baconii & Ebab200745 & Calamagrostis villosa & L78270 & AF231196 \\
\hline E. brachyelytri & Ebrc200752 & Brachyelytrum erectum & L78271 & AF231197 \\
\hline E. bromicola & Ebod200750 & Br. erectus & L78290 & AF231203 \\
\hline E. clarkii & EHIh200742 & H. lanatus & L78281 & AF231206 \\
\hline E. elymi & Eele200850 & Elymus virginicus & L78273 & AF231208 \\
\hline E. festucae & Efef90661 & F. rubra subsp. rubra & L06955 & AF231210 \\
\hline E. festucae & ATCC 201550 & F. rubra subsp. commutata & L06957 & AF231211 \\
\hline E. glyceriae & Eglg200747 & Glyceria striata & L78275 & AF231216 \\
\hline E. sylvatica & Esys200748 & Brachypodium sylvaticum & L78278 & AF231218 \\
\hline E. typhina & EAoh200738 & Anthoxanthum odoratum & L78288 & AF231222 \\
\hline E. typhina & EBps200739 & Brachypodium pinnatum & L78292 & AF231223 \\
\hline E. typhina & EDgh200740 & Dactylis glomerata & L78274 & AF231225 \\
\hline E. typhina & ELph200736 & Lolium perenne subsp. perenne & X52616 & AF231220 \\
\hline E. typhina & EPh200851 & Phleum pratense & L78280 & AF231226 \\
\hline E. typhina & EPn201667 & Poa nemoralis & AF062429 & AF231229 \\
\hline E. typhina & EPpn201669 & Poa pratensis & L78284 & AF231231 \\
\hline E. typhina & EPsh201666 & Poa silvicola & L78285 & AF231228 \\
\hline Epichloë sp. & EspHm9924 & Holcus mollis & AF457469 & AF457507 \\
\hline N. aotearoae & $\underline{\text { Naotear829 }}$ & Echinopogon ovatus & AF323370 & AF323391 \\
\hline Neotyphodium sp. & NspEhy1095 & El. hystrix & AF457479 & AF457519 \\
\hline N. huerfanum & $\underline{\text { Nhuer64040 }}$ & Festuca arizonica & AF457493 & AF457537 \\
\hline$\underline{N . \text { inebrians }}$ & $\underline{\text { Ninebrq818 }}$ & Ac. inebrians & AF457495 & AF457539 \\
\hline$\underline{\text { N. Iolii }}$ & $\underline{\text { NloLp135 }}$ & L. perenne subsp. perenne & L78286 & AF457540 \\
\hline N. typhinum var. canariense & $\underline{\text { NtcLedn989 }}$ & L. perenne subsp. canariense & AF176266 & AF457543 \\
\hline BbeTG-1 & $\underline{\mathrm{NBbd} 201559}$ & Bromus benekenii & AF250737 & AF231205 \\
\hline BpuTG-1 & $\underline{\text { NspBpu1081 }}$ & Bromus purgans & AF457477 & AF457517 \\
\hline EviTG-1 & $\underline{\text { NspEvr1087 }}$ & El. virvginicus & AF457478 & AF457518 \\
\hline$\underline{\text { HeuTG-1 }}$ & $\underline{\mathrm{NspHeu} 362}$ & Hordelymus europaeus & AF457488 & AF457528 \\
\hline$\underline{\mathrm{HbrTG}-1}$ & $\underline{\mathrm{NspHbr} 3635}$ & Hordeum brevisubulatum & AY137612 & AF532922 \\
\hline BsfTG-1 & $\underline{\text { NsBs } 420}$ & Bromus setifolis & AY707694 & AY707651 \\
\hline PsyTG-1 & NspPsy1097 & Poa sylvestris & AY163832 & AY166647 \\
\hline
\end{tabular}

* asexual species are underlined

used permutation; i.e. we compared the survival times of asexual lineages with those of 'asexual lineages' in trees in which we randomly assigned presence or absence of a sexual cycle to the extant species. If asexual lineages are not shorter-lived than sexual lineages, survival times of the real asexual lineages should be similar to those of the 'asexual lineages' in the permutation trees, because the latter 'asexual lineages' are in reality a mix of sexual and asexual lineages.
The phylogenetic trees were generated from $t u b B$ and tef $A$ sequences of 13 asexual and 20 sexual species (Table 1). Because hybrid species are asexual but have undergone at least one recombination event, they were excluded from the current analysis. Phylogenetic trees can be inaccurate in displaying phylogenetic relationships; often there is no tree that perfectly reflects the relationships, and often several alternative trees display relationships equally well; using only one single tree 
for this analysis could lead to misleading results. We therefore used software that generates a large number of trees and retains trees in proportion to how well they fit the data (see Methods), to generate 2600 trees based on each gene. Figure 2 shows a consensus network (Holland et al. 2005) in which conflicts in the relationships between species in multiple trees are highlighted, by showing alternative possible branching orders occurring in more than $10 \%$ of all trees. Such conflicts were rare and limited in the trees we generated.

Figure 3 shows the average survival times (as number of nucleotide substitutions) of asexual lineages in the tef $A$ trees (Fig. $3 a)$ and the $t u b B$ trees (Fig. 3b) as a box and whisker plot, and the distribution of average survival times of 'asexual lineages' in 100 sets of permutations; i.e., survival times calculated after randomly assigning absence of a sexual cycle to extant species

Figure 1 Inferring reproductive mode of ancestors (S: sexual; $A$ : asexual) and calculating the average age (in number of nucleotide substitutions) of the most ancient asexual ancestor of the asexual species (labelled A) in a tree containing both asexual and sexual (labelled $\mathrm{S}$ ) extant species. Ancestors at branch-points in the tree are considered to be sexual unless they only have asexual progeny (see results for details). The ages of the most ancient asexual ancestor of each the five asexual species are shown at the bottom. Note that in cases where the most ancient branch-point with an asexual ancestor is separated from a more ancient branch-point with a sexual ancestor by more than one nucleotide substitution, the age the most ancient ancestor is considered to have arisen half way between these two ancestors. For instance the age of the most ancient asexual ancestor of the asexual extant species on the far right is considered to be 3 substitutions, half way to the branchpoint with the sexual ancestor (6 substitutions). The average age of the most ancient ancestor of the asexual species in the Figure is $2.8(14 / 5)$. used (the ratio of asexual to sexual species was kept identical to that in the original data set). In both cases the survival times of the real asexual lineages were outside the range of survival times of 'asexual lineages' in permutated trees. Since the latter survival times include sexual species, we can conclude that asexual endophyte species survive for significantly shorter times than sexual species.

\section{Discussion}

Our data support the notion that absence of a sexual cycle, while possibly providing a short-term advantage, decreases the chance of survival in the long run, as has been observed in many other species (Rice 2002). We found no overlap between the survival time of asexuals and that of the species randomly selected in the

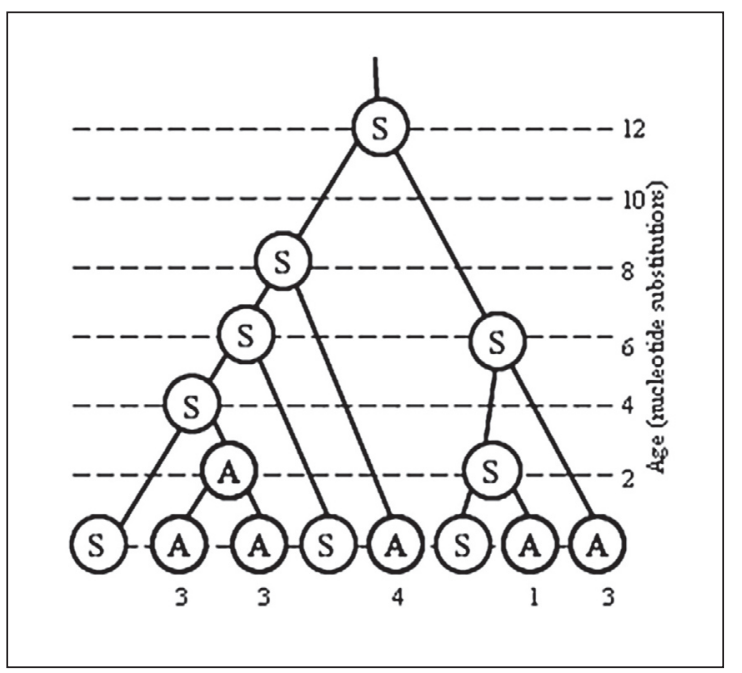

Figure 2 Consensus networks (Holland et al. 2005) of the 2600 trees for tefA (a) and tubB (b). Consensus networks were created using SplitsTree4 (Huson \& Bryant 2006) with a threshold value of 0.1 . Where the network has a simple branching structure it means that there are no alternative branches that appear in more than $10 \%$ of the trees. Where multiple possible branching orders (boxlike structures) are displayed it means that groups of trees disagree about the branching order.

(a)

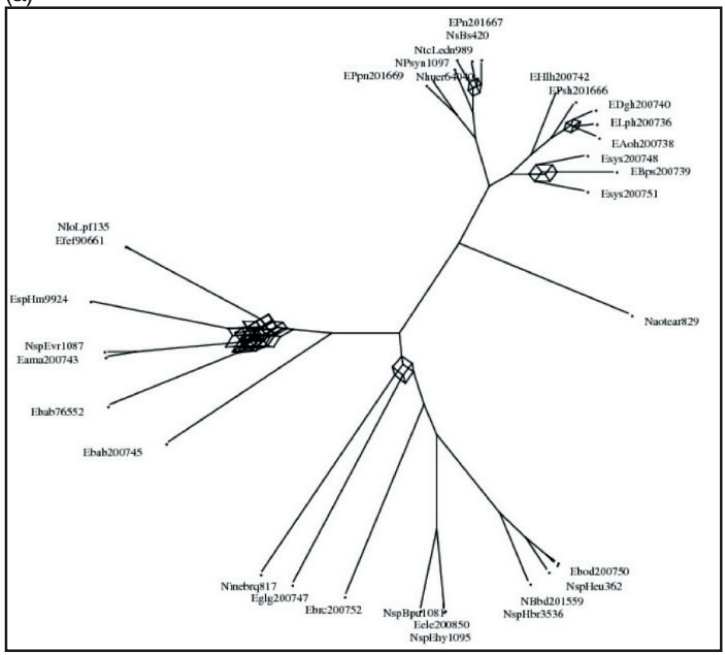

(b)

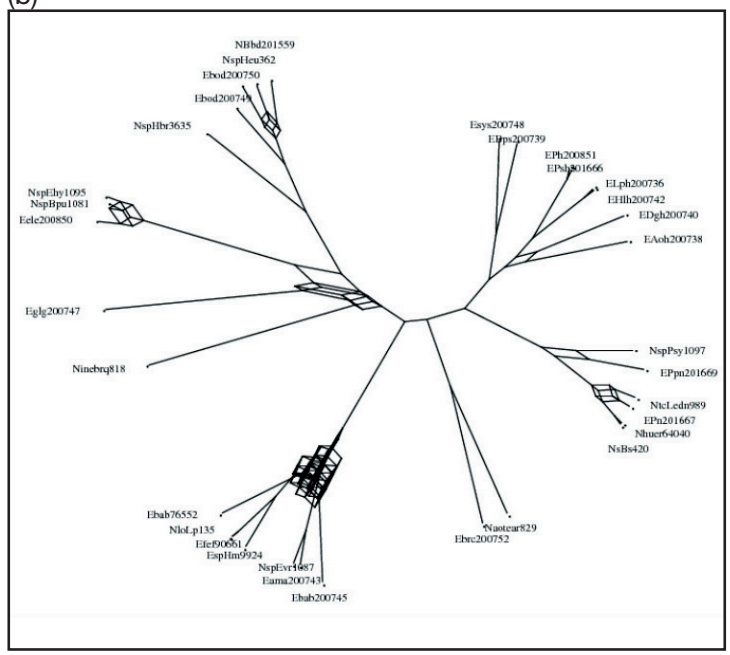


Figure 3 Histograms of ages of the most ancient ancestors of permutation-generated 'asexual species' and box and whisker representation of the ages of the oldest ancestors of the real extant asexual species in 2600 trees generated from tefA (a) and in 2600 trees generated from $t u b B(b)$ sequences.

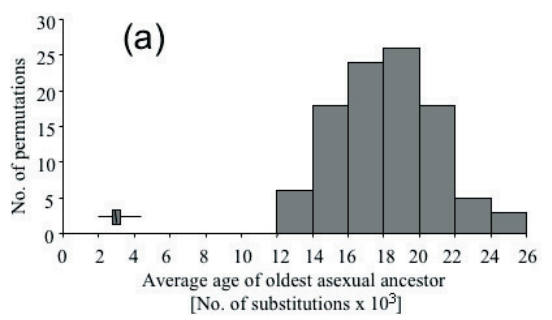

permutation test. However, there are a number of factors, which could affect our results. Firstly there are different algorithms that can be used to generate trees and times of divergence, and other algorithms would have given different times and trees. Secondly the trees reflect only the evolutionary histories of $t u b B$ and tef $A$ genes rather than of entire genomes. The trees are thus imperfect indicators of species age. Thirdly sampling bias could influence our results. Finally, different rates of nucleotide substitution in sexual and asexual species this would also affect the results. If clonal lineages had lower rates then we would underestimate their survival time. We can currently not exclude that these problems might affect our analysis. For the first and second factor there is no good reason to believe that they would act in a way so as to bias our analysis sufficiently so as to alter the relative differences between asexual and sexual lineage survival times. Future work will determine the possible magnitude of the bias caused by the third and fourth factors.

The exclusion of hybrid species is a shortcoming of the present analysis, which we hope to overcome in the future, because non-hybrid asexual endophytes are the exception rather than the norm (Moon et al. 2004). It must be borne in mind though that our assessment of non-hybrid status of a species is based on a very small number of genes, and it is conceivable that some of the asexual species we analysed are indeed hybrids. If so, the survival times of asexual lineages in our analysis may include some lineages that have undergone some exchange of genetic material. The fact that we nevertheless find a shorter survival time for asexual species strengthens the conclusion that exchange of genetic material is important for survival of endophyte lineages.

\section{ACKNOWLEDGEMENT}

J.S. was supported by a Massey University overseas leave grant.

\section{REFERENCES}

Carlile, M.; Watkinson, S.; Gooday, G. 2001. Genetic variation

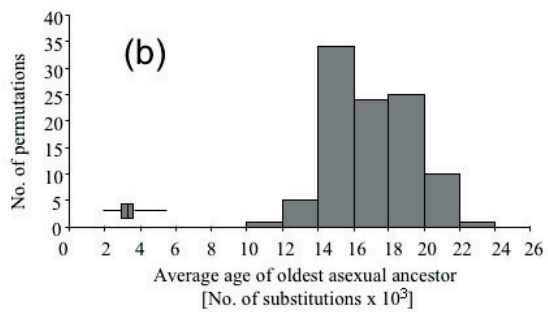

and evolution pp. 245-296. The fungi, 2 ed. Academic Press, San Diego, U.S.A.

Gentile, A.; Rossi, M.S.; Cabral, D.; Craven, K.D.; Schardl, C.L. 2005. Origin, divergence, and phylogeny of epichloe endophytes of native Argentine grasses. Molecular Phylogenetics and Evolution. 35: 196-208.

Goddard, M.R.; Godfray, H.C.; Burt, A. 2005. Sex increases the efficacy of natural selection in experimental yeast populations. Nature 434: 636-40.

Holland, B.R.; Delsuc, F.; Moulton, V. 2005. Visualizing conflicting hypotheses in large collections of trees using consensus networks. Systematic Biology 54: 66-76.

Huson, D.H.; Bryant, D. 2006. Application of phylogenetic networks in evolutionary studies. Molecular Biology and Evolution 23: 254-267.

Maynard Smith, J.; Smith, N. H., O’Rourke, M. Spratt, B.G. 1993. How clonal are bacteria? Proceedings of the National Academy of Sciences (USA) 90: 4334-4336.

Moon, C.D.; Craven, K.D.; Leuchtmann, A.; Clement, S.L.; Schardl, C.L. 2004. Prevalence of interspecific hybrids amongst asexual fungal endophytes of grasses. Molecular Ecology 13: 1455-1467.

Otto, S.P.; Lenormand, T. 2002. Resolving the paradox of sex and recombination. Nature Reviews Genetics 3: 252-261.

Purvis, A.; Hector, A. 2000. Getting the measure of biodiversity. Nature 405: 212-219.

Rice, W.R. 2002. Experimental tests of the adaptive significance of sexual recombination. Nature Reviews Genetics 3: 241-251.

Tibayrenc, M. 1999. Toward an integrated genetic epidemiology of parasitic protozoa and other pathogens. Annual Review of Genetics 33: 449-477.

Welch, D.M.; Meselson, M. 2000. Evidence for the evolution of bdelloid rotifers without sexual reproduction or genetic exchange. Science 288: 1211-1215. 